\title{
Clean Water Network Design for Refugee Camps
}

\author{
Özlem Karsu ${ }^{1} \cdot$ Bahar Y. Kara $^{1}$ (D) . Elif Akkaya ${ }^{1} \cdot$ Aysu Ozel $^{1}$
}

Accepted: 15 December 2020 / Pulished online: 13 January 2021

(C) The Author(s), under exclusive licence to Springer Science+Business Media, LLC part of Springer Nature 2021

\begin{abstract}
Motivated by the recent rise in need for refugee camps, we address one of the key infrastructural problems in the establishment process: The clean water network design problem. We formulate the problem as a biobjective integer programming problem and determine the locations of the water source, water distribution units and the overall network design (pipelines), considering the objectives of minimizing cost (total network length) and maximizing accessibility (total walking distance) simultaneously. We solve the resulting model using exact and heuristic approaches that find the set (or a subset) of Pareto solutions and a set of approximate Pareto solutions, respectively. We demonstrate the applicability of our approach on a real-life problem in Gaziantep refugee camp and provide a detailed comparison of the solution approaches. The novel biobjective approach we propose will help the decision makers to make more informed design decisions in refugee camps, considering the trade-off between the two key criteria of cost and accessibility.
\end{abstract}

Keywords Location - Covering · Water distribution network design · Biobjective optimization · Covering problem · Refugee camp design · Epsilon-constraint approach · NSGA2

\footnotetext{
Bahar Y. Kara

bkara@bilkent.edu.tr

Özlem Karsu

ozlemkarsu@bilkent.edu.tr

Elif Akkaya

elif.akkaya1991@gmail.com

Aysu Ozel

aysu.ozel@bilkent.edu.tr
}

1 Department of Industrial Engineering, Bilkent University, 06800, Ankara, Turkey 


\section{Introduction}

Post-disaster operations and related activities differ based on the nature and duration of the disasters (Holguín-Veras et al. 2012). For sudden onset disasters such as earthquakes, meeting urgent needs, reaching victims as soon as possible and handling the scarce resources under chaotic conditions are crucial. Therefore, mainly provisional solutions are found to respond to the urgency faster for shorter periods. For instance, shelter sites to be established after an earthquake are aimed to serve approximately one year. Thus, long-term infrastructures such as clean and drain water networks are not built, instead temporary solutions such as distribution of drinkable water and installation of toilet containers are implemented in these sites. On the contrary, recovery of long term disasters require more stable solutions which will respond to the needs for longer periods. Shelter sites to be established after a long term disaster necessitate additional and more durable features including infrastructure for clean and drain water. Due to the challenging conditions that the nature of disasters bring, effective planning of the available resources and operational efficiency are vital for long term solutions.

The refugee crisis is one of the challenges of today's world as a harsh long term disaster. It requires several actions that should be taken by countries providing asylum in co-operation with the Non-Governmental Organizations. One of the most important of those actions is establishing refugee camps in order to provide sheltering for refugees. Since the problems causing refugees to flee from their countries may remain for long periods, refugee camps may have to serve for many years. In the history, there are several examples of refugee camps that provide service for more than 20 years such as camps established in Bosnia and Herzegovina, Sudan and Afghanistan (UNHCR 2016). Therefore, refugee camps require long-term infrastructural planning.

The purpose of this study is addressing the clean water distribution network design problem in refugee camps. The paper is organized as follows. In Section 2, the clean water network design problem for refugee camps is defined and the related literature is presented. Then, a bi-objective mathematical model is given and exact and heuristic solution methodologies are stated in Sections 3 and 4, respectively. In Section 5, a case study with real life data is presented including computational results. The paper ends with concluding remarks.

\section{Problem Definition and Related Literature}

The long lasting nature of refugee crises necessitates detailed planning of the infrastructure of refugee camps to be established. One of the substantial resources that should be provided for refugees is water. Due to the long-servicing characteristic of the camps, water distribution network should be designed and it should fulfill the requirements that United Nations High Commissioner for Refugees (UNHCR 2007) or The sphere project (2011) has stated.

Water distribution in the camp can be achieved in different ways based on the local infrastructure and underground water availability. If a local water network is 
available, water can be provided by extending it and this can be considered as an urban network design/extension problem. If the local infrastructure is not accessible from the refugee camp or not sufficient, and there is no spring water at a reachable distance, then drilling wells is the only choice as a water source for the camp. In that case, the problem can be considered as a stand alone network design problem including its own source and distribution decisions. For instance, in the refugee camps in Turkey, water need is met with local water supply, since the local water network is expandable. But in Somalia refugee camp (TRC 2015), water is supplied by wells, as there is no local water network accessible and the region is arid. In both cases, the underlying OR problem is a specialized network flow problem including additional constraints due to the application dynamics.

Water network design in refugee camps is quite different from public water network design. In a public water network, water should be accessible from each demand point. Contrarily, in refugee camps, there should not necessarily be a water tap in every tent. In this case, water can be supplied to common use areas such as kitchens, showers, toilets and to the additional water taps for common use of collection of tents. For instance, in a camp in Somalia that serves internally displaced people, water requirement is met with common use water taps (TRC 2015) and there is one shared kitchen, whereas a kitchen and water are provided in each shelter in the Syrian refugee camp in Kilis, Turkey (The new york times magazine 2014).

In this study, we consider a water distribution network design problem for refugee camps. We study the case in which water source is a water well. Note that, a water well can be drilled only on a point where underground water exists and drilling is possible. Therefore, when well is used as the source, candidate locations for the well should be determined according to the underground water availability information. The network consists of a water well, water distribution units (WDUs) and pipelines connecting WDUs. In the context of our problem, WDU represents all types of water distribution points such as water taps, kitchens, showers and toilets. The main decisions are where to drill the well, where to locate WDUs and how to connect them.

While locating WDUs along the camp, one should make sure that the residents of each tent will have access to a WDU. Accessibility is an important concern in various domains and depending on the specifics of the problem it may be defined with respect to different resources or opportunities such as health care, drinking water or jobs (see Bielik et al. (2018) and the references therein). Here, access means ensuring that the distance between a tent and its closest WDU is below a certain threshold. There are various sources in the literature which define thresholds for access to clean water, Handbook for Emergencies and The Sphere Project Handbook are among the mostly used ones. Threshold distance is stated as 100 meter in Handbook for Emergencies by UNHCR (2007) and 500 meters in The Sphere Project Handbook (The sphere project 2011).

In this paper, we construct a bi-objective mathematical model for the clean water network design problem. The model aims to optimize two criteria, which are the construction cost of the network and walking distance of the users. We assume that the cost is directly proportional to the pipeline length, and ignore fixed costs if there are any. This issue, however, could be handled by modifying the model we provided and is considered as a future research topic. We define walking distance as the total 
walking distance of the refugees from shelters to the nearest WDU. The first criterion aims cost efficiency and the second one aims convenience for refugees to access water.

A substantial body of literature is devoted to the urban water network design problem in which decisions regarding the physical features of pipelines such as pipe length and radius are made (see D'Ambrosio et al. (2015) for a survey on mathematical programming approaches to drinking water distribution network optimization problems). Alperovits and Shamir (1977) and Eiger et al. (1994) develop systems that determine the pipe length and radius for a given water distribution network design. There are some other studies using heuristic methods such as simulated annealing and harmonic search for similar water network design problems (Cunha and Sousa (1999), Eusuff and Lansey (2003), Geem (2006), and Geem (2009)).

Farmani et al. (2005) approach the water network design problem from a multi criteria point of view. They use cost and utilization as their decision criteria and solve the same problem of finding the pipe length and radius using two multi criteria evolutionary algorithms which are non-dominated sorting genetic algorithm (NSGA) (Deb et al. 2002) and strong pareto evolutionary algorithm (SPEA), then compare the obtained results. Tanyimboh et al. (2010) use two different methods to design urban water network. They develop a multi-objective genetic algorithm and a stochastic search program which uses the concepts of evolution and a heuristic method. Maier et al. (2014) use evolutionary algorithms and other meta-heuristics to solve multi criteria optimization problems related to water resources systems. Iancu et al. (2006) analyze the trade-off between network length and cost criteria in water network design.

In the studies mentioned above, models and solution methodologies are used for finding the optimal pipe diameter and length, mostly for the given network of cities (some examples are New York and Hanoi). In such distribution systems, layout is pre-established. The main difference between the studies conducted in the literature and our work is the fact that network design of cities are predetermined i.e., the locations of both supply and demand nodes and the placement of pipes are known and the only concern is deciding on the specifications of the pipes, namely radius and length. In our problem, we do not have such a predetermined network, indeed we aim to determine the network design. However, in this study, we do not consider the physics of the flow and the required pipe settings. Specifically, we do not consider hydraulic parameters such as water pressure, water flow rate and water friction. Instead, while designing a water distribution network, we focus more on the requirements and dynamics of shelter sites for refugees.

Different from the studies mentioned previously, Nolz et al. (2010) propose a location-routing model for planning water distribution tours in disaster relief, in which there is no established water distribution network. The described problem aims to find optimal tours, travel modes and population nodes to be visited while optimizing two objectives. The first objective is minimizing a weighted sum of facility location criterion and maximal covering location criterion. A population node is considered to be covered if it is possible to reach a water distribution point within 2 hours travel time. The second objective is minimizing cost, measured in terms of travel time. They consider heterogeneous modes of transportation and different road 
categories e.g., damaged roads. Considering the problem requirements and multiple objectives stated, a decision support system is developed using a multi-objective meta-heuristic algorithm, NSGA-II. Although, the study of Nolz et al. (2010) and this study both use the coverage concern, the problems addressed are significantly different. First of all, in our problem we have to cover all demand nodes, which is guaranteed by covering constraints while in Nolz et al. (2010), all population center nodes are not necessarily covered (one of their objectives is minimizing the number of population center nodes remained uncovered). Nolz et al. (2010) plan water distribution tours (routes), we, however, design the water distribution network.

To the best of our knowledge, there are no studies that find the optimal clean water network design for a refugee camp while determining the locations of water source and WDUs. Our study considers minimal requirements and two objectives, and thus offers an important contribution to the literature.

\section{Model Development}

The water network design problem for refugee camps aims to locate a water well, WDUs and the pipelines to ensure the distribution of water among these components. In order to address the problem, a bi-objective mixed integer linear programming model is formulated given the locations of settlements, any predetermined WDU locations (such as kitchens, WCs), maximum number of WDUs that can be located, threshold distance for access of refugees and alternative well locations based on the underground water network.

When designing the water network, various objectives can be taken into consideration. As our focus is water network design for refugee camps, the decision makers should consider water accessibility of refugees in addition to the common concern in network design problem, which is cost. Thus, in this problem two objectives are utilized. The first objective is minimizing construction cost defined as the total pipe length and the second one is minimizing the total distance from each residential point to its closest WDU. With these objectives we intend to provide refugees with reasonably easy access to water.

In the stated problem, the locations of the well, WDUs and pipelines are aimed to be decided, so that the water distribution network can be designed. In that respect, the problem is similar to facility location and network design problems. Since water need not be available at tents in a refugee camp, WDUs will have to be located within the camp on specific spots such that all tents are covered within the threshold distance (UNHCR (2007) and The sphere project (2011)), as in set covering problems (Farahani et al. (2012) and Hakimi (1965) Also see Curtin et al. (2010) and Drezner and Drezner (2019) for some recent applications and extensions of the basic cover model). However there are some notable differences between the set covering and the clean water network design problem that we consider.

In the set covering problem, locations of facilities are determined, but the underlying distribution network infrastructure is assumed to exist. However, in the proposed water network design problem, the pipes between wells and WDUs will also be determined. Another difference between these two problems is the objective functions. In 
the set covering problem (Hakimi 1965), the objective is minimizing the total number of located facilities. However, in our refugee camp water network design problem, the maximum number of facilities that can be located is bounded by a constraint and the objectives are minimizing cost (total network length) and minimizing total walking distance of refugees to the nearest WDU.

We provide the bi-objective mathematical model that we propose, with the objectives of minimum total network length and total walking distance.

The following nomenclature will be used throughout the paper:

\section{Sets}

$\mathcal{I}=\{0,1,2, \ldots, n\}:$ the set of water demand points, and set of locations that a WDU can be established

$S=$ set of candidate water well locations, $S \subset \mathcal{I}$ (since a WDU is assumed to be established at the well location)

\section{Parameters}

$d_{i j}:$ the distance between two points $i \in \mathcal{I}$ and $j \in \mathcal{I}$

$p$ : maximum number of WDUs to be established

$\gamma$ : maximum distance allowed between a demand point and its closest WDU

$a_{i j}= \begin{cases}1, & \text { if } d_{i j} \leq \gamma \\ 0, & \text { otherwise }\end{cases}$

\section{Decision Variables}

$w_{s}= \begin{cases}1, & \text { if water well is drilled at point } s ; s \in S \\ 0, & \text { otherwise }\end{cases}$

$y_{i}= \begin{cases}1, & \text { if a WDU is installed at } i, i \in \mathcal{I} \\ 0, & \text { otherwise }\end{cases}$

$z_{i j}= \begin{cases}1, & \left.\text { if a pipe is installed between points } i \text { and } j ; i, j \in \mathcal{I} \text { (if } x_{i j}>0\right) \\ 0, & \text { otherwise }\end{cases}$

$u_{i j}= \begin{cases}1, & \text { if WDU at point } i \text { covers demand point } j ; i, j \in \mathcal{I} \\ 0, & \text { otherwise }\end{cases}$

$x_{i j}=$ total number of WDUs after link $(i, j), i \in \mathcal{I}, j \in \mathcal{I}$

We assume that the set of demand points $\mathcal{I}$ also represents the set of locations that a WDU can be established. If applicable, one can also define a set of specific locations at which a WDU should be established such as kitchens and bathrooms. The location of the well will be selected from the set of candidate locations $S$, which is determined in advance based on the underground water information. Decision variable $w_{s}$ is defined to choose the location of the water well among the elements of set $S$. It is assumed that all candidate water wells have the same characteristics e.g., water supply capacity. Decision variable $y_{i}$ shows whether a WDU is located at point $i$ or not. It is assumed that a WDU is also available at the location of well. Variable 
$z_{i j}$ is used to determine between which WDUs the pipelines should be installed and shows the network design. Decision variable $u_{i j}$ shows which WDUs can serve which demand points. Variable $x_{i j}$ is defined for connectivity between points $i$ and $j$ and it represents the total flow between these points.

Considering the requirements of the problem and its structural characteristics, the following bi-objective model is constructed.

$$
\begin{aligned}
& \text { Minimize } f_{1}=\sum_{i \in \mathcal{I}} \sum_{j \in \mathcal{I}} d_{i j} \cdot u_{i j} \\
& \text { Minimize } f_{2}=\sum_{i \in \mathcal{I}} \sum_{j \in \mathcal{I}} d_{i j} \cdot z_{i j} \\
& \text { Subject to } \\
& \begin{array}{l}
\sum_{i \in \mathcal{I}} a_{i j} \cdot y_{i} \geq \\
\sum_{i \in \mathcal{I}} y_{i} \leq p
\end{array} \\
& \sum_{i \in \mathcal{I}} x_{i k}-\sum_{i \in \mathcal{I}} x_{k i} \geq y_{k}-p . w_{k} \quad \forall k \in \mathcal{I}, k \neq i \\
& \sum_{i \in S} w_{s}=1 \\
& \sum_{i \in \mathcal{I} / S} w_{s}=0 \\
& 0 \leq x_{i j} \leq p \cdot z_{i j} \\
& \forall i, j \in \mathcal{I} \\
& 0 \leq x_{i j} \leq p \cdot\left(y_{j}+w_{j}\right) \\
& \forall i, j \in \mathcal{I} \\
& \sum_{i \in \mathcal{I}} u_{i j} \geq 1 \\
& \forall j \in \mathcal{I} \\
& u_{i j} \leq y_{i} \\
& \forall i, j \in \mathcal{I} \\
& w_{s}, y_{i}, z_{i j}, u_{i j} \in\{0,1\} \\
& \forall i, j \in \mathcal{I}, s \in S \\
& x_{i j} \text { integer }
\end{aligned}
$$

The objective functions are minimizing total walking distance of refugees and minimizing total network length calculated as total pipe length. Constraint Eq. 1 is the mandatory closeness constraint and it ensures that the distance between each residential point and its closest WDU is less than or equal to accessibility threshold $\gamma$. Constraint Eq. 2 guarantees that the total number of WDUs is less than or equal to p. Constraints Eqs. 3, 4 and 5 guarantee the flow balance of the network considering location of the well. For the case where there is no well $\left(w_{s}=0\right)$, constraint Eq. 3 ensures the difference between in and out flows, when there is a well, the constraint just becomes a non-active valid inequality.

Constraints Eqs. 4 and 5 ensure that a water well can only be drilled in one of the candidate well locations in $S$. Constraint Eq. 6 guarantees that if there is no pipe installed between $i$ and $j$, then there cannot be any flow from $i$ to $j$. Since the flow 
on any pipe cannot be more than $p$, this is a big $M$ type linearization with $p$ working as $M$. Constraint Eq. 7 provides the relation between the decision variables $y_{j}, w_{j}$ and $x_{i j}$. This constraint implies that if there is pipe and flow between points $i$ and $j$, there should be either well or WDU located at point $j$. Constraint Eq. 8 represents the coverage of each demand point $j$ with its closest WDU. Constraint Eq. 9 stands for connectivity of decision variables $y_{i}$ and $u_{i j}$, since it is not possible to cover any demand point from a node $i$ without establishing a WDU at that node. Constraints Eqs. 10 and 11 are the domain constraints.

Although the problem can be modeled by using each of the objective functions separately, the results obtained from those single objective programming models do not address the problem in the way we intend. For instance, when the only objective is minimization of total network length, WDUs are located very close to each other and clustered at a certain area in order to make the network as short as possible. In such a design, while some refugees are very close to WDUs, even having more than one WDU in a very short distance, majority of the refugees have their closest WDU at a distance of $\gamma$ (the threshold for access). When the objective function is minimization of total walking distance of refugees, water network is designed as a star network, meaning that for each individual WDU, there is a different pipe installed from the well, which is costly. Between these two extreme solutions, there are other Pareto optimal ones, which can only be found considering the two objectives simultaneously in a biobjective programming model as we propose. We intend to present the decision makers the entire set of Pareto optimal solutions for them to observe the trade-off between the two concerns of accessibility and cost. The decision maker can implement any Pareto solution that she chooses to provide more convenient access to water for refugees while still considering the cost aspect.

\section{Solution Methodologies and Performance Measures}

In this section, we first describe the solution methods we used for the above biobjective programming problem. We propose using the $\varepsilon$-constraint method to obtain the exact Pareto set for small to medium sized problems and heuristic algorithms for obtaining an approximate Pareto set for large-sized problems. We then explain performance measures utilized for assessing the quality of heuristic solutions.

\section{1 $\varepsilon$-Constraint Method}

To find the exact Pareto solutions of the model, we use epsilon constraint method (Haimes et al. 1971). In this method, one of the objectives is used as the objective function of the model, whereas the second one is iteratively bounded in a constraint. At each iteration, the bound on the second objective is decreased by a step size and the model is re-solved. Iterations are repeated in this manner, until the corresponding model becomes infeasible.

Without loss of generality, we take the first objective as total walking distance of refugees and the second objective as total network length denoted by $f_{1}$ and $f_{2}$, respectively. 
Let $f_{2}^{U B}$ be an upper bound on the second objective value, $N S$ be the set of nondominated solutions found so far, and $k$ be a predetermined step size parameter. Steps of the $\varepsilon$-constraint method are as follows:

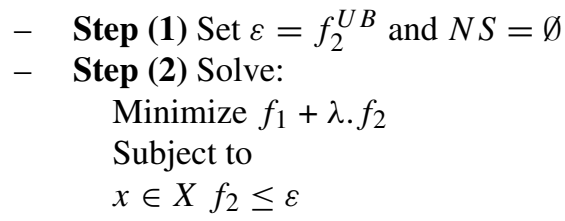

If the model is infeasible, go to Step (3). Otherwise, let the optimal objective function vector be $\left(f_{1}^{*}, f_{2}^{*}\right)$. $N S=N S \cup\left(f_{1}^{*}, f_{2}^{*}\right)$. Set $\varepsilon=f_{2}^{*}-k$ and repeat Step (2).

- Step (3) Stop and return NS.

Here, $x$ is the decision variable vector and $X$ is the feasible region of the corresponding problem. $\lambda$ is a parameter that is used to avoid weakly non-dominated but dominated solutions. In other words, when minimizing the first objective $\left(f_{1}\right)$, among the solutions that minimize $f_{1}$ the one that gives the minimum value for the second objective $\left(f_{2}\right)$ should be selected. To ensure this, in the objective function, the second objective is multiplied by the coefficient $\lambda$ which should be selected small enough in order not to head off the first objective. In our calculations, we set $\lambda$ to 0.001 , which is sufficiently small due to orders of magnitude.

If the second objective function could take only integer values, setting the step size $k$ to 1 would guarantee that the algorithm returns all Pareto solutions. However, in our problem, the distance matrix does not consist of integer values meaning that the second objective can take continuous values. In our computational analysis, we set $k$ to 0.1 .

Whenever possible, we find the whole Pareto set using this approach. However, since we observe that the single objective models become hard to solve for small $\gamma$ values, we also use this approach as a heuristic employing a time limit. The reason why the problem becomes harder to solve as $\gamma$ gets smaller is that when $\gamma$ is decreased, more WDUs should be used in order to satisfy the accessibility threshold constraint. Since the locations of additional WDUs and new connections between WDUs lead to different network designs, when the number of WDUs increases, the number of solution combinations also increases and feasible solution set builds up. This leads to higher computational times.

Note that one could also choose the minimizing network length as the main objective and gradually restrict the total walking distance in a constraint. However, the mathematical models become more difficult to solve when more emphasis is given to the network length. This is because, minimizing network length objective requires focusing on locating both WDUs and pipelines, i.e. it concentrates on the design of the whole network, which makes the problem more complicated. On the other hand, minimizing total walking distance leads to relatively easier-to-solve models as it mainly focuses on determining the locations of WDUs, not putting much emphasis on the locations of pipelines except connecting WDUs. We also observe this in our 
preliminary experiments, hence use total walking distance as the main objective in our computational analysis.

\subsection{Metaheuristic Algorithms}

To find the approximate Pareto solutions, we use two metaheuristic algorithms. The first algorithm used is Non-Dominated Sorting Genetic Algorithm for MultiObjective Optimization (NSGA-II) proposed by Deb et al. (2002).

The MOEA Framework source code of the NSGA-II algorithm is modified taking the problem specifications into account (MOEA 2016). For the working principle of the algorithm, see Fig. 1. As it is seen in the figure, Prim's algorithm is used for finding the optimal total network lengths for a given set of candidate solutions, since it finds the optimal network tree (Ahuja et al. 1993).

The adapted NSGA-II algorithm is solved for each candidate well location in $S$. A pool of solutions that are obtained for each candidate location are gathered and the non-dominated solutions are selected among those.

The second metaheuristic algorithm used is an improved version of the Strength Pareto Evolutionary Algorithm (SPEA2) proposed by Zitzler et al. (2001). Similar to the NSGA-II algorithm, the MOEA Framework source code of the SPEA2 algorithm

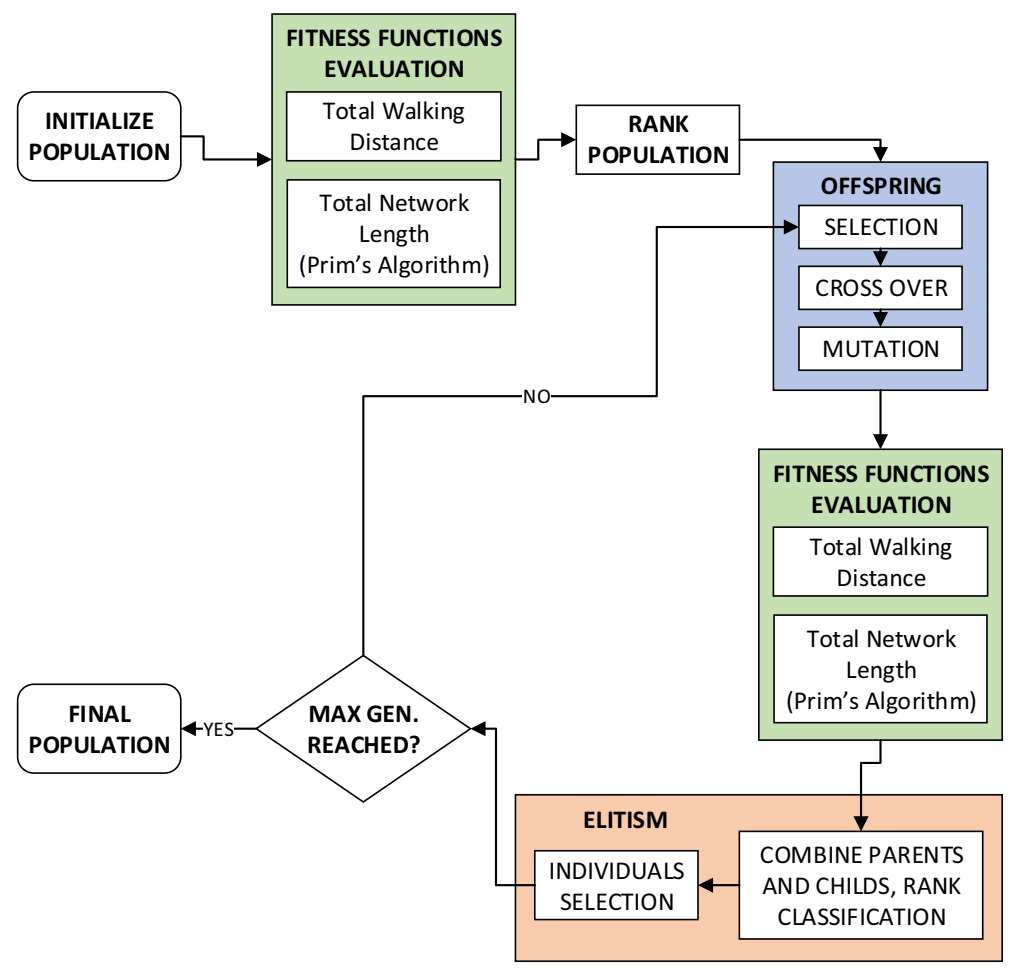

Fig. 1 Working principle of adapted NSGA-II algorithm 
is modified taking the problem specifications into account (MOEA 2016). That is, Prim's algorithm is used for finding the optimal total network lengths for a given set of candidate solutions and SPEA2 algorithm is solved for each candidate well location in $S$. The working principle of the algorithm can be seen in Fig. 2.

As we will discuss in Section 5.1 in detail, in the computational analysis on small scale problems, we observe that SPEA2 algorithm requires significantly more time to solve the problem. Thus for larger problems we only utilized NSGA-II algorithm.

\subsection{Performance Measures}

Performance measures are used in order to assess the quality of the solutions returned by the NSGA-II and SPEA2 algorithms. Whenever possible, we compare the results of the metaheuristic algorithms with the exact Pareto set using three performance measures. The first performance measure shows the percentage of exact Pareto solutions found by the metaheuristic algorithm and the others show the average and maximum distances from the exact Pareto frontier.

- $P \%$ : Ratio of nondominated objective vectors returned by the metaheuristic algorithm;

$$
P \%=\frac{|A N S \cap N S|}{|N S|}
$$

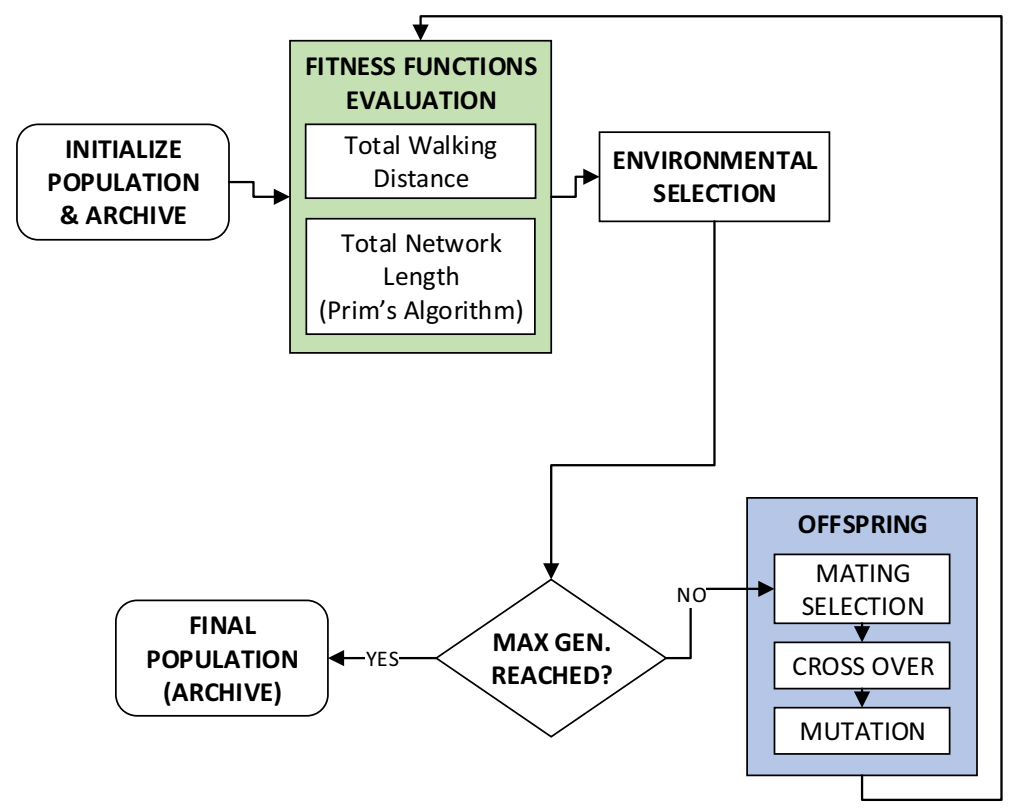

Fig. 2 Working principle of SPEA2 algorithm 
where NS represents the exact set of nondominated objective vectors and ANS represents the set of nondominated objective vectors returned by metaheuristic algorithm. Note that, in the remainder of the paper, the sets of nondominated objective vectors returned by NSGA-II and SPEA2 are distinguished as $\mathrm{ANS}_{1}$ and $\mathrm{ANS}_{2}$, respectively.

In order to consider the closeness of the approximate solutions to their nondominated counterparts, we make use of two distance measures. To state these measures, we assume that $\left(f_{1}^{r}, f_{2}^{r}\right)$ is in set NS and $\left(f_{1}^{q}, f_{2}^{q}\right)$ is in set ANS, then we calculate the following values:

$$
\begin{aligned}
& R_{1}=\max _{\left(f_{1}^{r}, f_{2}^{r}\right) \in N S} f_{1}^{r}-\min _{\left(f_{1}^{r}, f_{2}^{r}\right) \in N S} f_{1}^{r} \quad \text { (range for the } f_{1} \text { values in set NS) } \\
& R_{2}=\max _{\left(f_{1}^{r}, f_{2}^{r}\right) \in N S} f_{2}^{r}-\min _{\left(f_{1}^{r}, f_{2}^{r}\right) \in N S} f_{2}^{r} \quad \text { (range for the } f_{2} \text { values in set NS) } \\
& R\left(\left(f_{1}^{r}, f_{2}^{r}\right),\left(f_{1}^{q}, f_{2}^{q}\right)\right)=\max \left\{0, \frac{1}{R_{1}} \cdot\left(f_{1}^{q}-f_{1}^{r}\right), \frac{1}{R_{2}} \cdot\left(f_{2}^{q}-f_{2}^{r}\right)\right\}
\end{aligned}
$$

Here, value of the function $R$ equals to zero, if solutions $q$ and $r$ are the same. Otherwise, $R$ takes the value of the maximum component-wise normalized distance between point $q$ and point $r$.

Using these functions, the following distance measures are defined:

- Distance1 (D1): The average distance between the points of set NS and the points in set ANS

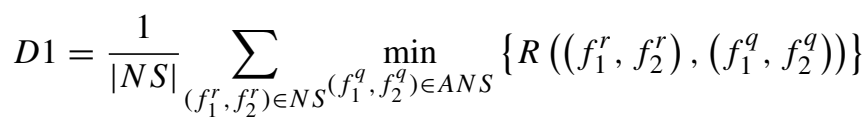

- Distance2 (D2): The maximum distance between the points of set NS and the points in set ANS

$$
D 2=\max _{\left(f_{1}^{r}, f_{2}^{r}\right) \in N S}\left\{\min _{\left(f_{1}^{q}, f_{2}^{q}\right) \in A N S}\left\{R\left(\left(f_{1}^{r}, f_{2}^{r}\right),\left(f_{1}^{q}, f_{2}^{q}\right)\right)\right\}\right\}
$$

The smaller the distance values are, the better the performance of the metaheuristic algorithm is.

For the instances in which finding the exact Pareto solutions is not possible in reasonable time, we set a time limit for each model that we solve in the epsilon constraint method and find solutions with gaps. Then, we compare the solutions returned by the metaheuristic algorithms with this set of approximate Pareto solutions. Since the reference set is also an approximate set, the performance measures that we use in such cases are different from the ones stated above. We utilize the following measures to check the performance of the set of solutions returned by the metaheuristic algorithms, which is denoted by ANS in comparison with the set of solutions returned by time limited epsilon constraint method, denoted by GNS. As the set of solutions returned by time limited epsilon constraint method (GNS) is an approximate set, we also utilize the same performance measures to check the quality of this set. 
- Spacing (SP): This metric shows the diversity of the solutions in a given solution set (ANS/GNS). Let $\left(f_{1}^{i}, f_{2}^{i}\right)$ and $\left(f_{1}^{j}, f_{2}^{j}\right)$ be two different solutions in a given set $\mathrm{A}$. SP is evaluated using the following equation:

$$
\begin{gathered}
S P=\sqrt{\frac{1}{n-1} \cdot \sum_{i=1}^{n}\left(d_{i}-\bar{d}\right)^{2}} \\
d_{i}=\min _{j}\left|f_{1}^{i}-f_{1}^{j}\right|+\left|f_{2}^{i}-f_{2}^{j}\right| \quad i, j=1, \ldots, n
\end{gathered}
$$

where $n$ is the number of solutions in the given set, $d_{i}$ is the minimum Manhattan distance between the solution $\left(f_{1}^{i}, f_{2}^{i}\right)$ and any other solution $\left(f_{1}^{j}, f_{2}^{j}\right)$ in the same set and $\bar{d}$ is the mean value of all $d_{i}$ s.

Smaller SP values indicate more uniformly distributed points, which is desirable.

- Maximum Spread (MS): This measure shows the maximum extension covered by the solution set (ANS/GNS) (Li and Zheng 2009). For a given solution set A, it is evaluated using the following equation:

$$
M S=\sqrt{\sum_{m=1}^{2}\left(\max _{\left(f_{1}, f_{2}\right) \in A} f_{m}-\min _{\left(f_{1}, f_{2}\right) \in A} f_{m}\right)^{2}}
$$

The larger MS value is, the better the solution set is, because higher MS value indicates that the solutions are more spread.

- Set coverage (SC): Let Set1 and Set2 be two sets of solution vectors. $\mathrm{SC}(\operatorname{Set} 1, \operatorname{Set} 2)$ is the ratio of points that are dominated (for two vectors $v$ and $t, t$ dominates $v$ when $t \leq v$ ) by at least one point in Set1.

$$
S C(\operatorname{Set} 1, \operatorname{Set} 2)=\frac{|\{v \in \operatorname{Set} 2 \mid \exists t \in \operatorname{Set} 1: t \leq v\}|}{|\operatorname{Set} 2|}
$$

SC(Set1,Set2) can take a value in [0,1] interval. Both SC(ANS,GNS) and $\mathrm{SC}(\mathrm{GNS}, \mathrm{ANS})$ values are calculated.

If points in ANS dominate all points in GNS, then by definition $\mathrm{SC}(\mathrm{ANS}, \mathrm{GNS})$ is 1 , while $\mathrm{SC}(\mathrm{ANS}, \mathrm{GNS})=0$ implies the opposite. In general, both SC(ANS, GNS) and SC(GNS, ANS) should be considered, as there can be intersections between sets. For instance, SC(ANS, GNS)=1 and SC(GNS, ANS $=0.5$ means ANS dominates all points in GNS (in the weak sense), but half of the vectors in GNS are the same with those of ANS. If SC(ANS, GNS) $=1$ and $\mathrm{SC}(\mathrm{GNS}, \mathrm{ANS})=0$, we can say that ANS is strictly better than GNS.

\section{Case Study: The Refugee Camp in Gaziantep, Turkey}

To test the behavior of the mathematical model and proposed solution methodologies, computational studies are performed. In these studies, data based on a refugee camp in Gaziantep, Turkey is used. The data to be used in the model includes distance 
between nodes, threshold value for access and candidate nodes for well locations. (The distances are actual distances between nodes in the Gaziantep refugee camp. We then created different instances by changing the threshold levels ( $\gamma$ 's) and trying alternative well locations.) Generation of the data sets and the instances created from this data are discussed below. (The data is also available from the authors upon request.)

The Gaziantep refugee camp is established by Turkish Red Crescent (TRC), following the layout standards of the Sphere Project.

According to minimum requirements of Sphere Project, the tents should be grouped with enough spaces in between, for fire breaks and transportation purposes. Based on those standards, we first formed the block structure of the Gaziantep Refugee Camp. We consider each housing cluster as a demand node in our network. With this assumption and the standards stated above, we create a distance matrix that shows the Euclidean distances between each pair of demand nodes. There are 73 housing clusters, namely nodes in Gaziantep refugee camp.

We use the standards of Handbook for emergencies by UNHCR (2007) and take accessibility threshold $(\gamma)$ values as 60, 80 and 100 meters in the computational studies. For determining the location of the well, we use different candidate well locations. Random sets of candidate well locations $(S)$ are generated with different cardinalities: $1,5,15,25$ respectively. In the special case where the cardinality of $S$ is equal to 1 , the location of the well is fixed.

In the computational analysis, the model is solved for two different layouts, one consisting of 38 nodes and the other one consisting of 73 nodes. For each cardinality of $S$ and accessibility threshold combination, 2 different sets of candidate well locations (S) is used and in total 24 instances are created for each layout. For the first layout consisting of 38 nodes, which is a subset of all nodes in Gaziantep refugee camp, the model is solved for each instance created using the $\varepsilon$-constraint approach, NSGA-II and SPEA2 algorithms, and the performance of NSGA-II and SPEA2 are assessed compared to the exact set of solutions, whenever possible. For the second layout considering all 73 nodes in Gaziantep refugee camp, due to the computational challenges, solutions are obtained using $\varepsilon$-constraint approach with a time limit and the NSGA-II algorithm. In the computational analysis, the mathematical models are solved using CPLEX 12 with default CPLEX parameter settings on a PC with Intel Core 2 Duo T6400 (2.0 GHz) processor and 4GB RAM.

\subsection{Results for $\mathbf{3 8}$ Nodes}

While solving models, different upper limits for the number of WDUs $(p)$ are defined for different $\gamma$ values. When $\gamma=60, p$ is set as 9. Likewise, when $\gamma$ is 80 and 100, $p$ is set as 6 , and 5 respectively. As $\gamma$ gets larger, we expect a decrease in the number WDUs that should be located, hence we decrease $p$. Besides, we ensured that at least one WDU is established in a common use kitchen and another one in a common use WC by fixing 2 WDU locations in the 38 node setting.

The corresponding models are solved with both $\varepsilon$-constraint method and NSGA-II algorithm. When $\gamma=80$ and 100, the exact Pareto solutions are found. When $\gamma=60$, each single objective model in the $\varepsilon$-constraint method is solved for 10 minutes and 
the resulting solutions are reported with corresponding optimality gaps. Then, the solutions obtained are assessed using the performance measures stated in Section 4.3.

Since the whole set of exact Pareto solutions can be found for $\gamma=80$ and 100, but only approximate Pareto solutions can be found for $\gamma=60$, they are discussed in different tables. Comparative summary of exact and heuristic solutions for the model with accessibility thresholds $\gamma=80$ and 100, and two different sets of candidate well locations (S) for each cardinality level is given in Table 1. In the table, \# of NS represents number of nondominated solutions returned by the epsilon-constraint method, \# of $\mathrm{ANS}_{1}$ represents number of approximate nondominated solutions returned by NSGA-II and \# of $\mathrm{ANS}_{2}$ represents number of approximate nondominated solutions returned by SPEA2. $S$ is the set of randomly selected candidate well locations and $|S|$ represents the cardinality of this set.

It can be observed from Table 1 that the solution time of the epsilon constraint approach increases as the number of candidate well locations increases, and the increase is more pronounced when $\gamma=80$. The solution time of NSGA-II linearly increases as cardinality of well set increases. This is because the NSGA-II algorithm is run $|S|$ times, each time fixing a different candidate as the well. The solution time of SPEA2 increases in a similar manner. However, SPEA2 takes much more longer time to solve the problem compared to NSGA-II and in most instances the solution times of SPEA2 are even longer than those of the epsilon constraint approach.

Table 1 Summary table for $\gamma=80$ and 100

\begin{tabular}{|c|c|c|c|c|c|c|c|c|}
\hline \multirow[b]{2}{*}{$\gamma$} & \multirow[b]{2}{*}{ Set } & \multirow[b]{2}{*}{ No } & \multicolumn{2}{|c|}{ Epsilon Constraint Approach } & \multicolumn{2}{|l|}{ NSGA-II } & \multicolumn{2}{|l|}{ SPEA2 } \\
\hline & & & \# of NS & Time(sec) & $\#$ of $\mathrm{ANS}_{1}$ & Time(sec) & $\#$ of $\mathrm{ANS}_{2}$ & Time(sec) \\
\hline \multirow[t]{8}{*}{80} & $|S|=1$ & 1 & 5 & 140 & 5 & 2 & 5 & 305 \\
\hline & & 2 & 14 & 917 & 14 & 2 & 14 & 108 \\
\hline & $|S|=5$ & 3 & 9 & 449 & 9 & 10 & 9 & 1449 \\
\hline & & 4 & 10 & 338 & 10 & 10 & 10 & 1163 \\
\hline & $|S|=15$ & 5 & 14 & 3001 & 14 & 30 & 14 & 3955 \\
\hline & & 6 & 13 & 1434 & 13 & 30 & 13 & 3712 \\
\hline & $|S|=25$ & 7 & 14 & 3310 & 14 & 50 & 14 & 6369 \\
\hline & & 8 & 14 & 3288 & 14 & 50 & 14 & 6233 \\
\hline \multirow[t]{8}{*}{100} & $|S|=1$ & 9 & 6 & 13 & 6 & 1 & 6 & 25 \\
\hline & & 10 & 12 & 87 & 12 & 1 & 12 & 18 \\
\hline & $|S|=5$ & 11 & 12 & 94 & 12 & 5 & 12 & 112 \\
\hline & & 12 & 17 & 913 & 17 & 5 & 17 & 110 \\
\hline & $|S|=15$ & 13 & 22 & 2174 & 21 & 15 & 21 & 400 \\
\hline & & 14 & 21 & 1610 & 21 & 15 & 21 & 326 \\
\hline & $|S|=25$ & 15 & 22 & 2530 & 22 & 25 & 22 & 638 \\
\hline & & 16 & 22 & 2355 & 22 & 25 & 22 & 561 \\
\hline
\end{tabular}


In order to assess the performance of NSGA-II and SPEA2, we use performance measures P\%, D1 and D2 that can be seen on Table 2. Note that both meta-heuristic algorithms return the same set of solutions, therefore we only report a single value for each of the related performance measure. Since the results were similar for different cardinality of well sets, we show the minimum, average and maximum values of $\mathrm{P} \%$, D1 and D2 for the instances with the same $\gamma$.

For $\gamma=80$ instances, both NSGA-II and SPEA2 find the whole set of exact Pareto solutions showing that the algorithms work very well for these instances. The results are similar for $\gamma=100$, average value of $\mathrm{P} \%$ is equal to 89.21 , indicating that most of the exact Pareto solutions are found in these instances by the meta-heuristic algorithms. As a result, the distance values are negligible. An exception occurs for one instance where $\mathrm{P} \%$ is around 13 , yet the maximum distances are small indicating that the approximate Pareto frontiers are close to the exact one. Although NSGA-II and SPEA2 perform similarly in terms of solution quality, NSGA-II may be preferable, since its solution times are significantly shorter than those of SPEA2.

Figure 3 illustrates the layout designs for different solutions on the Pareto Frontier of an instance where $\gamma=80$ and $|S|=5$. More specifically, candidate well locations are restricted to the set $\{1,7,11,16,22\}$ and the locations of two fixed WDUs are nodes 15 and 28, as they are the common kitchen and WC in the camp. Figure 3a represents the solution with the minimum walking distance value 1289.8 , which also has the longest network length: 387.55 . Figure $3 \mathrm{~b}$ shows an intermediate solution, where total walking distance is 1347.8 and the network length is 338.46. Finally, Fig. $3 \mathrm{c}$ represents the solution with the total walking distance 1470.8 and the minimum network length value 302.07. It can be observed from Fig. 3 that the network design changes in favor of network length as one moves from Fig. 3a to Fig. 3c. This is a direct result of the epsilon constraint algorithm starting with minimizing total walking distance edge of the Pareto frontier.

For $\gamma=60$ instances, we report the minimum, average and maximum number of solutions found and solution times in Table 3. Each single objective model in the epsilon constraint approach is solved for 10 minutes and the solutions are reported with the corresponding optimality gaps. In Table 3, \# of GNS denotes the number of approximate nondominated solutions with gaps returned by the $\varepsilon$-constraint method. Similarly, \# of $\mathrm{ANS}_{1}$ and \# of $\mathrm{ANS}_{2}$ are the number of approximate nondominated solutions returned by the NSGAII and SPEA2 algorithms, respectively. In Table 3, we do not categorize the solutions by the cardinality of well sets as we do in Table 1 for $\gamma=80$ and 100 since we have not observed a significant change in \# of GNS, \# of ANS and solution times for different cardinality levels.

Table 2 Performance measures for NSGA-II and SPEA2 with $\gamma=80$ and 100

\begin{tabular}{|c|c|c|c|c|c|c|c|c|c|}
\hline \multirow[b]{2}{*}{$\gamma$} & \multicolumn{3}{|l|}{$\mathrm{P} \%$} & \multicolumn{3}{|l|}{ D1 } & \multicolumn{3}{|l|}{ D2 } \\
\hline & Min & Avg & $\operatorname{Max}$ & Min & Avg & Max & Min & Avg & Max \\
\hline 80 & 100 & 100 & 100 & 0 & 0 & 0 & 0 & 0 & 0 \\
\hline 100 & 13.64 & 89.21 & 100 & 0 & 0.0103 & 0.0826 & 0 & 0.0224 & 0.1791 \\
\hline
\end{tabular}




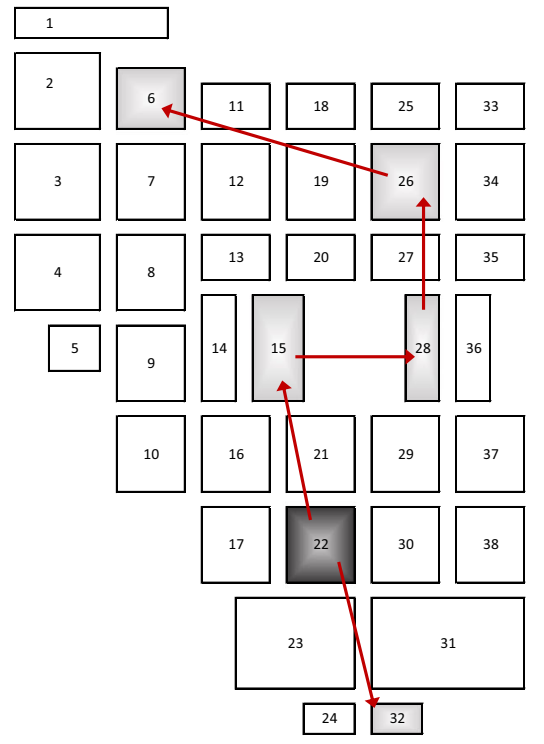

(a)

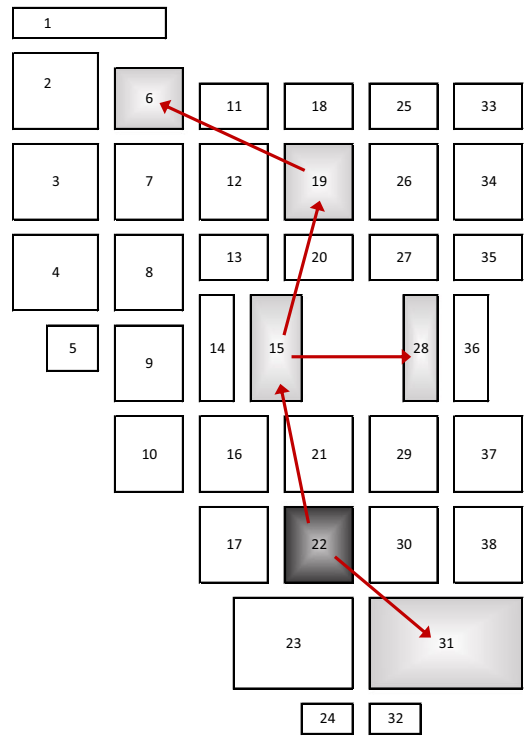

(b)

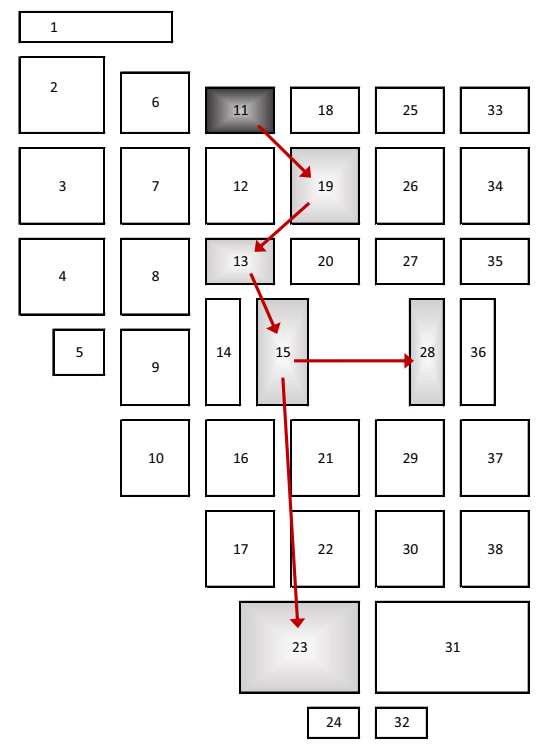

(c)

Fig. $3 \gamma=80, S=\{1,7,11,16,22\}$. The minimum total walking a. An intermediate solution $\mathbf{b}$. The minimum network length $\mathbf{c}$

As seen in Table 3, the number of solutions returned by the epsilon-constraint approach (GNS) is quite less than the number of solutions returned by the NSGAII and SPEA2. This is because, to obtain GNS, the single objective models in the 
Table 3 Summary table with $\gamma=60$

\begin{tabular}{|c|c|c|c|c|c|c|c|c|c|}
\hline & \multicolumn{3}{|c|}{$\begin{array}{l}\text { Epsilon Constraint } \\
\text { Approach (with gap) }\end{array}$} & \multicolumn{3}{|c|}{ NSGA-II } & \multicolumn{3}{|c|}{ SPEA2 } \\
\hline & Min & Avg & $\operatorname{Max}$ & Min & Avg & $\operatorname{Max}$ & Min & Avg & $\operatorname{Max}$ \\
\hline$\#$ of GNS/ANS $1 / \mathrm{ANS}_{2}$ & 6 & 8.3 & 12 & 22 & 23.1 & 27 & 23 & 23.5 & 27 \\
\hline CPU Time (sec) & 1998 & 3865.9 & 6081 & 3 & 35.5 & 75 & 92 & 1208.88 & 2660 \\
\hline
\end{tabular}

$\varepsilon$-constraint approach are solved for ten minutes. If this time limit is exceeded and no integer solution is found, $\varepsilon$-constraint algorithm stops. Although there are fewer solutions in epsilon constraint method, solution times are considerably longer than those of NSGA-II.

To assess the quality of the solutions returned by metaheuristic algorithms and epsilon constraint method with gap, we use SP, MS and SC metrics. The performance measures can be seen on Table 4. Recall that the less the SP value is and contrarily the more the MS value is, the better the solution set is. Therefore, both NSGA-II and SPEA2 outperform $\varepsilon$-constraint approach with respect to spacing (SP) and spread (MS). This is because of the early termination of the $\varepsilon$-constraint algorithm due to the time limit. However, part of the solutions returned by the NSGA-II and SPEA2 are dominated by the ones returned by the epsilon constraint approach, as seen in the $\mathrm{SC}\left(\mathrm{GNS}_{\mathrm{ANS}}\right)$ and $\mathrm{SC}\left(\mathrm{GNS}, \mathrm{ANS}_{2}\right)$ metrics. We observe that if the epsilon constraint approach can find a solution within the time limit, it is better than the ones returned by the heuristic algorithms in the same part of the Pareto frontier. However, epsilon constraint approach explores only a part of the Pareto frontier, failing to find solutions in the minimum total network length part of the frontier, while metaheuristic algorithms offer solutions representing that part. As it can be seen from Table 4, NSGA-II and SPEA2 perform similarly with respect to all performance measures. In fact, $\mathrm{SC}\left(\mathrm{ANS}_{1}, \mathrm{ANS}_{2}\right)$ and $\mathrm{SC}\left(\mathrm{ANS}_{2}, \mathrm{ANS}_{1}\right)$ measures indicate that on the average $96 \%$ of the SPEA2 solutions are the same with those of NSGA-II.

Figure 4 shows the solutions for an instance with $|S|=1$ and $\gamma=60$, where $\mathrm{SC}\left(\mathrm{ANS}_{1}, \mathrm{ANS}_{2}\right)$ is 0.87 . In this chart, GNS found by $\varepsilon$-constraint method, $\mathrm{ANS}_{1}$ found by NSGA-II and $\mathrm{ANS}_{2}$ found by SPEA2 are shown. It is seen that NSGA-II and SPEA2 offer more solutions but some of them are dominated by the solutions found by the epsilon constraint solutions. Note that, $\mathrm{ANS}_{1}$ and $\mathrm{ANS}_{2}$ solutions are more spread and also represent the minimum total network length edge of the frontier while the GNS solutions are clustered at a certain area, the minimizing total walking distance edge of the frontier. Therefore, the solutions in GNS are not able to capture the entire spectrum of the Pareto frontier.

While finding solutions, the epsilon constraint algorithm starts with minimizing total walking distance edge and the first iterations are solved in relatively shorter times compared to further ones. After a few iterations, as we approach to the minimum network length side of the Pareto front, the solution time of the models increases significantly and no integer solution is found in a given time limit, thus the algorithm stops. 
Table 4 Performance measures for NSGA-II and SPEA2 with $\gamma=60$

\begin{tabular}{|c|c|c|c|c|c|c|c|c|c|}
\hline \multirow[b]{2}{*}{ Measures } & \multicolumn{3}{|c|}{$\begin{array}{l}\text { Epsilon Constraint } \\
\text { Approach (with gap) }\end{array}$} & \multicolumn{3}{|c|}{ NSGA-II } & \multicolumn{3}{|l|}{ SPEA2 } \\
\hline & Min & Avg & $\operatorname{Max}$ & Min & Avg & Max & Min & Avg & Max \\
\hline SP & 3.39 & 7.16 & 11.28 & 3.02 & 4.81 & 10.35 & 3.15 & 5.29 & 10.00 \\
\hline MS & 64.08 & 89.48 & 128.45 & 128.85 & 149.10 & 187.26 & 128.85 & 152.22 & 187.26 \\
\hline $\mathrm{SC}\left(\mathrm{GNS}, \mathrm{ANS}_{1}\right)$ & 0.50 & 0.67 & 1.00 & & & & & & \\
\hline $\mathrm{SC}\left(\mathrm{GNS}, \mathrm{ANS}_{2}\right)$ & 0.52 & 0.68 & 1.00 & & & & & & \\
\hline $\mathrm{SC}\left(\mathrm{ANS}_{1}, \mathrm{GNS}\right)$ & & & & 0.00 & 0.10 & 0.25 & & & \\
\hline $\mathrm{SC}\left(\mathrm{ANS}_{1}, \mathrm{ANS}_{2}\right)$ & & & & 0.87 & 0.96 & 1.00 & & & \\
\hline $\mathrm{SC}\left(\mathrm{ANS}_{2}, \mathrm{GNS}\right)$ & & & & & & & 0.00 & 0.10 & 0.25 \\
\hline $\mathrm{SC}\left(\mathrm{ANS}_{2}, \mathrm{ANS}_{1}\right)$ & & & & & & & 0.91 & 0.99 & 1.00 \\
\hline
\end{tabular}

As it can be seen in Fig. 4, even for the instance having the lowest $\mathrm{SC}\left(\mathrm{ANS}_{1}, \mathrm{ANS}_{2}\right)$ value, i.e. the instance in which $\mathrm{ANS}_{1}$ has the worst quality compared to $\mathrm{ANS}_{2}$, the quality difference between the two solution sets is not high. That is, NSGA-II and SPEA2 perform similarly in terms of solution quality. However, the solution times of SPEA2 are considerably higher than NSGA-II even for the small scale problems. Therefore, we use NSGA-II algorithm in our computational analysis for larger problem instances having 73 nodes.

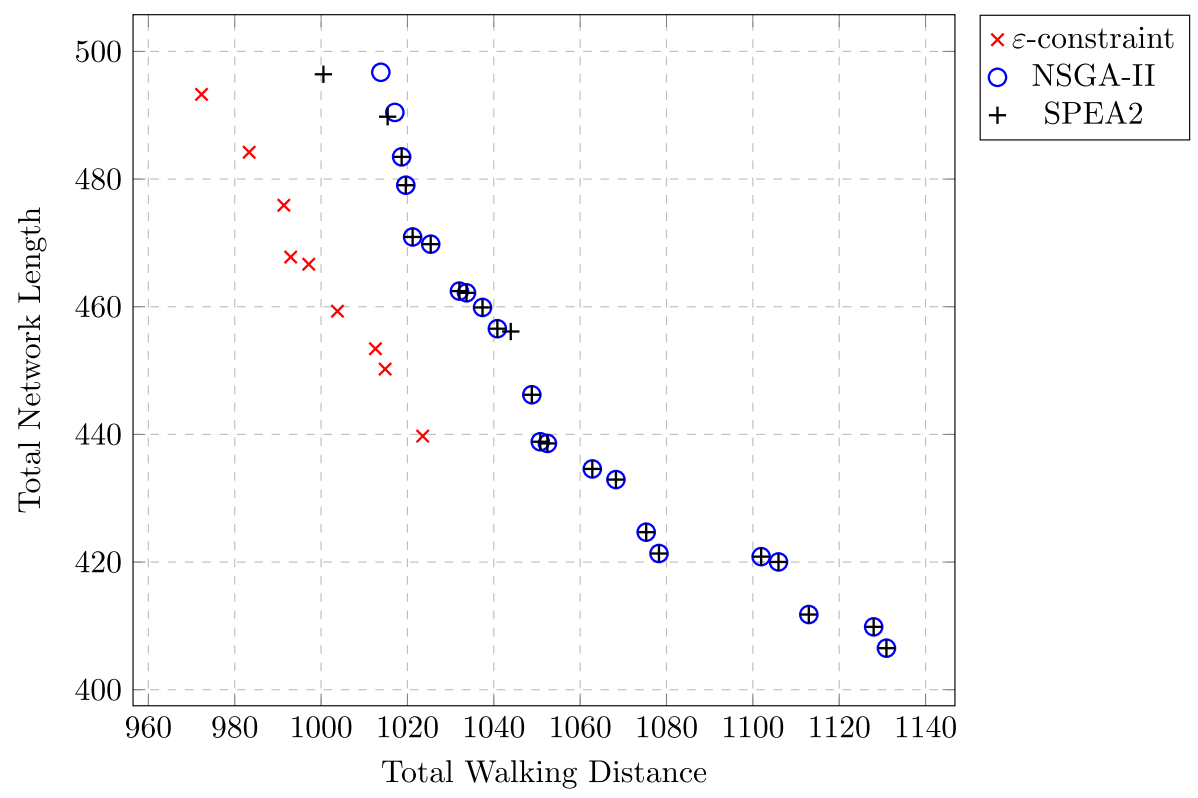

Fig. 4 Comparison of NS, $\mathrm{ANS}_{1}$ and $\mathrm{ANS}_{2}$ for an instance of $\gamma=60,|S|=1$ 


\subsection{Results for 73 Nodes}

Based on the preliminary studies, it is observed that for larger problem instances, it is harder to obtain exact solutions with the $\varepsilon$-constraint method in reasonable time. Since it is shown in the previous part that NSGA-II algorithm performs considerably well, we solve the larger models with 73 nodes using NSGA-II. We also solve these instances with $\varepsilon$-constraint approach with a time limit. We consider the same $\gamma$ values as 60,80 and 100 . Since number of nodes are approximately doubled, the upper limit for WDUs $(p)$ is also increased. We set $p$ as 20, 12 and 8 for $\gamma=60,80$ and 100 respectively. Similar to the 38-node setting, we fix the locations of some WDUs, in this case 4 of them, with the same assumption that they are common use kitchens and WCs whose locations are predetermined. 2 instances for each cardinality level of the

Table 5 Summary table for $\gamma=60,80$ and 100

\begin{tabular}{|c|c|c|c|c|c|c|}
\hline \multirow[b]{2}{*}{$\gamma$} & \multirow[b]{2}{*}{ Set } & \multirow[b]{2}{*}{ No } & \multicolumn{2}{|c|}{$\begin{array}{l}\text { Epsilon Constraint } \\
\text { Approach (with gap) }\end{array}$} & \multicolumn{2}{|l|}{ NSGA-II } \\
\hline & & & \# of GNS & CPU Time (sec) & \# of ANS & CPU Time (sec) \\
\hline \multirow[t]{8}{*}{60} & $|S|=1$ & 1 & 1 & 827 & 31 & 45 \\
\hline & & 2 & 1 & 715 & 37 & 55 \\
\hline & $|S|=5$ & 3 & 1 & 650 & 132 & 225 \\
\hline & & 4 & 1 & 753 & 66 & 246 \\
\hline & $|S|=15$ & 5 & 1 & 820 & 82 & 717 \\
\hline & & 6 & 1 & 763 & 163 & 675 \\
\hline & $|S|=25$ & 7 & 1 & 617 & 97 & 1133 \\
\hline & & 8 & 1 & 779 & 171 & 1116 \\
\hline \multirow[t]{8}{*}{80} & $|S|=1$ & 9 & 2 & 676 & 100 & 48 \\
\hline & & 10 & 2 & 667 & 13 & 52 \\
\hline & $|S|=5$ & 11 & 1 & 610 & 110 & 248 \\
\hline & & 12 & 2 & 689 & 110 & 252 \\
\hline & $|S|=15$ & 13 & 1 & 614 & 123 & 627 \\
\hline & & 14 & 1 & 69 & 115 & 631 \\
\hline & $|S|=25$ & 15 & 2 & 703 & 129 & 1238 \\
\hline & & 16 & 1 & 606 & 126 & 1254 \\
\hline \multirow[t]{8}{*}{100} & $|S|=1$ & 17 & 8 & 1930 & 6 & 46 \\
\hline & & 18 & 6 & 1250 & 9 & 34 \\
\hline & $|S|=5$ & 19 & 7 & 2214 & 10 & 202 \\
\hline & & 20 & 6 & 2201 & 7 & 237 \\
\hline & $|S|=15$ & 21 & 7 & 2510 & 8 & 648 \\
\hline & & 22 & 7 & 1403 & 12 & 693 \\
\hline & $|S|=25$ & 23 & 7 & 2218 & 39 & 1225 \\
\hline & & 24 & 7 & 2840 & 12 & 1097 \\
\hline
\end{tabular}


Table 6 Performance measures for NSGA-II

\begin{tabular}{|c|c|c|c|c|c|c|c|}
\hline \multirow[b]{2}{*}{$\gamma$} & \multirow[b]{2}{*}{ Measures } & \multicolumn{3}{|c|}{$\begin{array}{l}\text { Epsilon Constraint } \\
\text { Approach (with gap) }\end{array}$} & \multicolumn{3}{|c|}{ NSGA-II } \\
\hline & & Min & Avg & Max & Min & Avg & Max \\
\hline \multirow[t]{4}{*}{60} & SP & - & - & - & 3.58 & 8.27 & 11.72 \\
\hline & MS & - & - & - & 287.51 & 1118.56 & 2334.90 \\
\hline & SC(GNS,ANS) & 0.00 & 0.00 & 0.00 & & & \\
\hline & SC(ANS,GNS) & & & & 0.00 & 0.00 & 0.00 \\
\hline \multirow[t]{4}{*}{80} & SP & - & - & - & 6.40 & 14.71 & 25.75 \\
\hline & MS & - & - & - & 212.81 & 1195.70 & 2190.70 \\
\hline & SC(GNS,ANS) & 0.00 & 0.02 & 0.09 & & & \\
\hline & SC(ANS,GNS) & & & & 0.00 & 0.13 & 1.00 \\
\hline \multirow[t]{4}{*}{100} & SP & 10.96 & 18.66 & 31.36 & 0.05 & 19.45 & 25.92 \\
\hline & MS & 59.63 & 135.42 & 207.71 & 207.71 & 486.04 & 1192.80 \\
\hline & SC(GNS,ANS) & 0.00 & 0.51 & 1.00 & & & \\
\hline & SC(ANS,GNS) & & & & 0.00 & 0.54 & 0.86 \\
\hline
\end{tabular}

well sets, in total 8 instances for each $\gamma$ value and overall 24 instances are created. In Table 5, detailed results for instances with accessibility thresholds $\gamma=60,80$ and 100, and two different sets of candidate well locations (S) for each cardinality are shown.

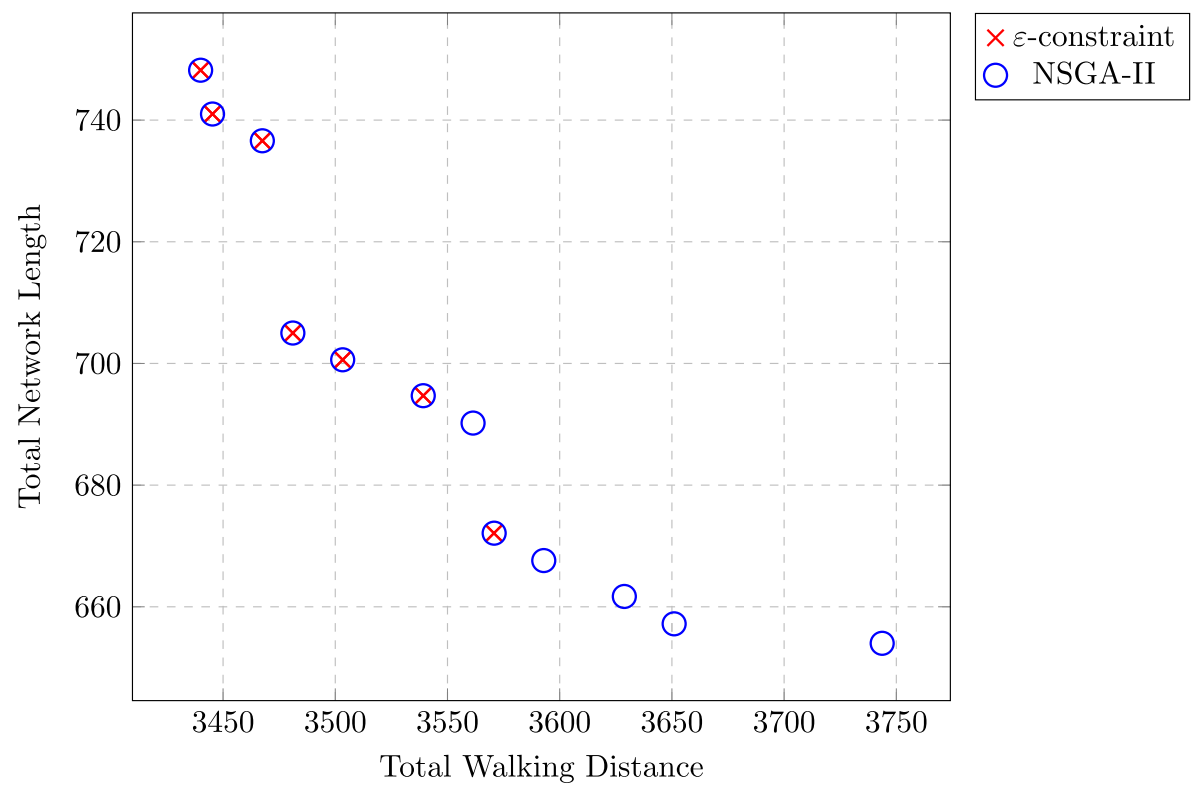

Fig. 5 Comparison of GNS and ANS for an instance of $\gamma=100,|S|=25$ 
As it can be seen from the Table 5, the epsilon constraint approach fails to find more than one solution in instances with small $\gamma$ values. Since both ANS and GNS contain approximate Pareto solutions, the number of solutions returned may not be a good indicator of quality. Therefore, we compare the solutions in GNS and ANS using SP, MS, and SC metrics and report the results in Table 6.

Note that for most of the instances with small $\gamma$ values, the epsilon constraint approach with time limit returns a single solution, hence SP and MS measures can not be calculated. It is seen that NSGA-II outperforms the epsilon constraint approach with gap with respect to spacing and spread. Moreover, the solutions are of comparable quality indicated by the set coverage metric: Both for $\gamma=80$ and $\gamma=100$, more solutions in set GNS are dominated by at least one point in ANS, than vice versa. We provide the sets for an example instance in Fig. 5.

\section{Conclusion}

In this study, we address the clean water network design problem in refugee camps and propose a bi-objective programming model that determines the locations of the water well, WDUs and designs the general network structure by taking relevant constraints such as threshold for access into account. To the best of our knowledge, our study offers the first problem definition, model and solution methods for clean water distribution network in refugee camps. Moreover, considering two relevant criteria of cost and accessibility simultaneously, the approach helps the decision makers to observe the trade-offs and make more informed design choices.

Exact and heuristic solution methodologies are proposed to solve the bi-objective models. In order to find the exact Pareto frontier, the epsilon constraint method is utilized. It is observed that for some instances, $\varepsilon$-constraint method takes considerably long time to solve. Therefore, NSGA-II algorithm is adapted to find the solutions in shorter times. Our computational experiments demonstrated that the algorithm returns good quality solutions.

In the cases where the exact Pareto set could not be found in reasonable time, we also use the epsilon constraint approach in a heuristic manner. We solve each single objective model in the epsilon constraint method with a gap and compare the corresponding results with those of the NSGA-II. It is observed that NSGA-II algorithm outperforms this heuristic version of the epsilon constraint method.

This study can be extended in several directions. One possible extension would be incorporating a fixed cost associated with the construction of the network, which is independent of the network length, into our model. Another extension would be considering hydraulic parameters and associated costs, which make water network design problems harder to solve. Another research direction worth exploring is considering other components of the water network such as water storage units, tanks and depots, which leads to more extensive problems. Finally, a third objective about the utilization of WDUs can be considered. Exact algorithms could be improved to solve larger problems in reasonable time or alternative heuristic solution methodologies can be implemented. 
Acknowledgements The authors are grateful to the anonymous reviewers and the editor for their valuable feedback on an earlier version of this paper that resulted in improved content and exposition.

\section{References}

Ahuja RK, Magnanti TL, Orlin JB (1993) Network flows: theory, algorithms and applications Alperovits E, Shamir U (1977) Design of optimal water distribution systems. Water Resour Res 13(6):885900

Bielik M, König R, Schneider S, Varoudis T (2018) Measuring the impact of street network configuration on the accessibility to people and walking attractors. Netw Spat Econ 18(3):657-676

Cunha MDC, Sousa J (1999) Water distribution network design optimization: simulated annealing approach. J Water Resour Plan Manag 125(4):215-221

Curtin KM, Hayslett-McCall K, Qiu F (2010) Determining optimal police patrol areas with maximal covering and backup covering location models. Netw Spat Econ 10(1):125-145

D'Ambrosio C, Lodi A, Wiese S, Bragalli C (2015) Mathematical programming techniques in water network optimization. Eur J Oper Res 243(3):774-788

Deb K, Pratap A, Agarwal S, Meyarivan T (2002) A fast and elitist multiobjective genetic algorithm: Nsga-ii. IEEE Trans Evol Comput 6(2):182-197

Drezner T, Drezner Z (2019) Cooperative cover of uniform demand. Netw Spat Econ 19(3):819-831

Eiger G, Shamir U, Ben-Tal A (1994) Optimal design of water distribution networks. Water Resour Res 30(9):2637-2646

Eusuff MM, Lansey KE (2003) Optimization of water distribution network design using the shuffled frog leaping algorithm. J Water Resour Plan Manag 129(3):210-225

Farahani RZ, Asgari N, Heidari N, Hosseininia M, Goh M (2012) Covering problems in facility location: a review. Comput Ind Eng 62(1):368-407

Farmani R, Savic D, Walters G (2005) Evolutionary multi-objective optimization in water distribution network design. Eng Optim 37(2):167-183

Geem ZW (2006) Optimal cost design of water distribution networks using harmony search. Eng Optim 38(03):259-277

Geem ZW (2009) Particle-swarm harmony search for water network design. Eng Optim 41(4):297-311

Haimes YY, Ladson L, DWismer A (1971) Bicriterion formulation of problems of integrated system identification and system optimization. IEEE Trans Syst Man Cybern 3:296

Hakimi SL (1965) Optimum distribution of switching centers in a communication network and some related graph theoretic problems. Oper Res 13(3):462-475

Holguín-Veras J, Jaller M, Van Wassenhove LN, Pérez N, Wachtendorf T (2012) On the unique features of post-disaster humanitarian logistics. J Oper Manag 30(7):494-506

Iancu P, Pleşu V, Lavric V (2006) Cost versus network length criteria in water network optimal design. Comput Aided Chem Eng 21:1821-1826

Li M, Zheng J (2009) Spread assessment for evolutionary multi-objective optimization. In: Ehrgott M, Fonseca CM, Gandibleux X, Hao J-K, Sevaux M (eds) Evolutionary Multi-Criterion Optimization. Springer, Berlin, pp x216-230

MOEA (2016) The MOEA framework: a free and open source java framework for multiobjective optimization [Online]. Available: http://www.moeaframework.org/Accessed:January, 2016

Maier HR, Kapelan Z, Kasprzyk J, Kollat J, Matott L, Cunha M, Dandy GC, Gibbs MS, Keedwell E, Marchi A et al (2014) Evolutionary algorithms and other metaheuristics in water resources: Current status, research challenges and future directions. Environ Model Softw 62:271-299

Nolz PC, Doerner KF, Hartl RF (2010) Water distribution in disaster relief. Int J Phys Distrib Logist Manag 40(8/9):693-708

TRC (2015) Reports of somalia humanitarian aid. [Online]. Available: https://www.kizilay.org.tr/Upload/ Dokuman/Dosya/03101983_-2015-somali-insani-yardim-operasyonu.pdfAccessed:June, 2016

Tanyimboh T, Ward K, Prasad T, Jarvis E, Kanyoza A (2010) Multiobjective optimization and multicriteria decision making for water networks. In: Proceedings of the computing and control in the water industry conference (CCWI 2009). University of Sheffield Sheffield, UK, pp 277283 
The new york times magazine (2014) How to build a perfect refugee camp. [Online]. Available: http:// www.nytimes.com/2014/02/16/magazine/how-to-build-a-perfect-refugee-camp.html?_r=1Accessed: June, 2016

The sphere project (2011) Humanitarian charter and minimum standards in humanitarian response. [Online]. Available:http://www.ifrc.org/PageFiles/95530/The-Sphere-Project-Handbook-20111. pdfAccessed:June, 2016

UNHCR (2007) Handbook for emergencies. [Online]. Available: http://www.ifrc.org/PageFiles/95884/D. 01.03.Accessed:June, 2016

UNHCR (2016) Bosnia and herzegovina regional office. [Online]. Available: http://reporting.unhcr.org/ node/12002\#_ga=1.221477905.865779010.1466775782 Accessed: June, 2016

Zitzler E, Laumanns M, Thiele L (2001) Spea2: Improving the strength pareto evolutionary algorithm. TIK-report, vol. 103

Publisher's Note Springer Nature remains neutral with regard to jurisdictional claims in published maps and institutional affiliations. 